\section{CHEMISTRY, PHARMACY, AND MATERIA MEDICA.}

\section{ON THE FORMATION OF A NATIONAL PHAR- MACOPGELA.}

\section{By Mr. Souire.}

AFTER the remarks published in our last $\mathrm{Mr}$. Squire brings into a tabular form all the tinctures of the three British pharmacopoias, from which it appears that the discrepancies in the preparation of these forms of medicine are innumerable, and must render a prescription extremely uncertain unless the intended tincture be accurately defined by appending its source. Mr. Squire continues:-

"I now propose directing attention to discrepancies of another kind, not existing among the formulce of the different Colleges, for preparations bearing the same name, but among formule all contained in the same Pharmacopoeia for preparations of the same class. In alluding to these, I shall confine myself to the London Pharmacopoeia. Thus, taking, for instance, the formulse for the diluted acids, we find no uniformity in their relative strengths or saturating powers.

"There appears, as far as I can judge, no reason why the liquid diluted acids should "not be all of one saturating power (the hydrocyanic being made the exception to the rule). If mixed in the following proportions:- three measures of sulphuric acid and thirty of water, three of nitric acid and fifteen of water, three of muriatic acid and five of water, they will be found to have severally about the same saturating power as our present diluted sulphuric acid. They might then be accurately adjusted, so that a fluid drachm should exactly saturate twenty-eight grains of crystallized carbonate of soda.

"The acetic and phosphoric acids, and any others that may at any future time be introduced into the Pharmacopoeia, should be brought to the same strength. The prescriber would thus be relieved from the trouble he must now take in distinguishing between the different strengths of this class of medicines, and the circumstance of the acids being tested as to their saturating power during the process of dilution, would ensure their uniformity.

"In considering how far it would be possible to introduce a similar uniformity of strength in other classes of medicines, I have especially directed my attention to the tinctures. "The following quotations from some of our most eminent writers on pharmaceutical subjects, will show that while there is a difference of opinion among them with reference to the value of these preparations in their present form, much of the objection urged against them would be removed if the strength of many of the tinctures were increased; and such increase would be necessary in order to reduce them to one uniform strength, or in other words to equalize the doses.

" ' With the exception of those which contain very active ingredients, tinctures are not important preparations, and though extensively cmployed, are not indispensable.'-Brande's Dictionary of Materia Medica, 1839.

" "The form of tincture is one of the best in Pharmacy; for the menstruum is a powerful solvent of the active constituents of drugs, it presents them in small volume, it preservés them very long unaltered, and it is for the most part a convenient medium for uniting them with other substances in extempore prescriptions.'-Christison's Dispensatory, 1842.

" 'Tinctures are not of very extensive use as remedies, except in cases where stimulants are indicated, the solvent even in cases of a few fluid drachms often acting more powerfully on the living system than the principles it holds in solution. When the action of a substance is the reverse of a stimulant, it cannot with propriety be exhibited in thi form, unless the dose be so small that the operation of the spirit cannot be taken into the account, as in tincture of foxglove, \&c.'-Thomson's Dispensatory, 1844.

" ' Tinctures are solutions of various substances in spirit of wine of different degrees of strength. The substances which are best adapted for tinctures are those which are active in small doses.'-Phillips's Translation of the London Pharmacopcia.

"By adopting the process of percolation in the preparation of tinctures, there would, of course, be no difficulty in effecting the object $I$ allude to, and it would only be necessary to fix upon any one tincture, such as that of opium, or of foxglove, as the standard, and to adjust the strength of all the others, so that their ordinary doses should cor- respond. On referring to Mr. Phillips's list of deses in his Translation of Pharmacopoia, I find that there are-

12 Tinctures, whose maximum dose is $\mathbf{f} 3 \mathbf{j}$.

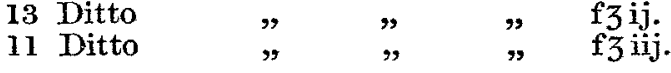

"There are three, namely, the compound tinctures of aloes, of rhubarb, and of senna, the doses of which exceed the above.

"The fluid alkaline preparations would form another class, the doses of which might be made to assimilate.

"I have ventured to make these suggestions for the consideration of those to whom is committed the preparation of our Pharmacopoeia, and I trust that the three Colleges will adopt by the commencement of the year 1850 one set of formulæ for the whole kingdom."-Pharmaceutical Jour., Nov.

PREPARATION OF MEDICTNAX CHLORIDE OF SODA.

"Mr. Payen has published a process by which he prepares this substance of uniform strength, without involving the trouble of first preparing chlorine gas. For this purpose he has recourse to the mutual action of carbonate of soda and chloride of lime. M. Payen first ascertained the mean proportion of chlorine in the medicinal solutions of M. Labarraque, and then compared it with the proportion of chlorine in chloride of lime, represented by $98^{\circ}$ of Gay Lussac's chronometer. These experiments showed that one part by weight of chloride of lime of $98^{\circ}$ was equivalent to 20 parts of medicinal chloride of soda; that 100 parts of this chloride of lime are completely decomposed by 138 parts of chrystallized sub-carbonate of soda; and, finally, that by adding 62 parts of the last salt to the neutral solution of the chloride, its excess was sufficient to render the compound permanent. The following is therefore the formula for the preparation of the chloride of soda:-

\begin{tabular}{lll} 
& \multicolumn{4}{c}{ Parts. } \\
Chloride of lime at $98^{\circ} \ldots \ldots \ldots \ldots \ldots . . . \ldots$ & 100 by weight. \\
Crystallized sub-carbonate of soda ... 138 & Do. \\
Water ..................................... 1800 & Do.
\end{tabular}

The chloride of lime is to be dissolved, and the sediment well washed; carbonate of soda dissolved by heat is to be poured into the solution, the precipitate allowed to subside, the clear fluid decanted, and the solid matter washed on a filter. The collected solutions are neutral chloride of soda; 62 parts of the sub-carbonate of soda are then to be dissolved in the remainder of the water and added to the preparation; the whole being then filtered, a limpid solution is obtained, indicating $5^{\circ}$ by the areometer of Baume. It is the pure chloride of soda. If the chloride of lime be of any other strength than $98^{\circ}$, then the relation is easily ascertained by the chlorometer and a brief calculation, and the equivalent of the quantity above directed must be used.-Bull. Philom., 1826, 151. From the Quarterly Journal of Science, Literature, and Art; from January to March, 1827. New Series.

[We reprint the $\mathrm{w}_{\mathrm{a}} \mathrm{above}$ at the request of a correspondent there is no doubt of the correctness of the process, and its results.-ED.]

\section{MEDICAL SOCHETY OE LONDON.}

MONDAY, KOV. 3, 1845.-DOR. THEOPIILCS THOMPSON, PRESIDENT.

$$
\text { TRACHEOTONT AND LARYAGOTOMY. }
$$

Mr. PIlcrer, in reference to the case detailed at the last meeting, said that he agreed with Mr. Robarts that the inflammation was of an erratic character, and that the seat of it, causing the more tormidable symptoms, was at the upper part of the rima glottidis, and that the chords vocales were involved. Regarding the operation as necessary, where should it be performed? Generally, laryngotomy was the least advisable proceeding, for the larynx was often the seat of the disease. Tracheotomy was better. There was certainly more fear of hæmorrhage from the external incisions, in tracheotomy, but this hrmorrhage could be arrested previously to the opening of the tube itself. This was not the case in laryngotomy, in which there was usually an artery wounded, the hæmorrhage from which might not be alwars so harmless in its consequences as in the present instance.

Mr. Robarts believed that, in his case, tracheotomy would have been the more advisable proceeding, as it could be performed at a spot further removed from the seat of disease; but he could not resist taking advantage of the facility which presented itself of opening the larynx. With regard to the artery, it would not be wounded in a transverse incision over the larynx, and a tube could be introduced through such an inci- 